\title{
Exploiting Flexibly Assignable Work to Improve Load Balance*
}

\author{
Ali Pınar \\ NERSC \\ Lawrence Berkeley National Laboratory \\ Berkeley, CA 94720 \\ apinar@lbl.gov
}

\author{
Bruce Hendrickson \\ Parallel Computing Sciences Department \\ Sandia National Laboratories \\ Albuquerque, NM 87185-1110 \\ bah@cs.sandia.gov
}

\begin{abstract}
In many applications of parallel computing, distribution of the data unambiguously implies distribution of work among processors. But there are exceptions where some tasks can be assigned to one of several processors without altering the total volume of communication. In this paper, we study the problem of exploiting this flexibility in assignment of tasks to improve load balance. We first model the problem in terms of network flow and use combinatorial techniques for its solution. Our parametric search algorithms use maximum flow algorithms for probing on a candidate optimal solution value. We describe two algorithms to solve the assignment problem with $\log W_{T}$ and $|P|$ probe calls, where $W_{T}$ and $|P|$, respectively, denote the total workload and number of processors. We also define augmenting paths and cuts for this problem, and show that any algorithm based on augmenting paths can be used to find an optimal solution for the task assignment problem. We then consider a continuous version of the problem, and formulate it as a linearly constrained optimization problem, i.e., $\min \|A x\|_{\infty}$, s.t. $B x=d$. To avoid solving an intractable $\infty$-norm optimization problem, we show that in this case minimizing the 2-norm is sufficient to minimize the $\infty$-norm, which reduces the problem to the well-studied linearly-constrained least squares problem. The continuous version of the problem has the advantage of being easily amenable to parallelization.
\end{abstract}

\footnotetext{
${ }^{\dagger}$ The author is also supported by the Director, Office of Science, Division of Mathematical, Information, and Computational Sciences of the U.S. Department of Energy under contract DE-AC03-76SF00098.

${ }^{*}$ This work was funded by the Applied Mathematical Sciences program, U.S. Department of Energy, Office of Energy Research and performed at Sandia, a multiprogram laboratory operated by Sandia Corporation, a Lockheed-Martin Company, for the U.S. DOE under contract number DEAC-94AL85000.
}

Permission to make digital or hard copies of all or part of this work for personal or classroom use is granted without fee provided that copies are not made or distributed for profit or commercial advantage and that copies bear this notice and the full citation on the first page. To copy otherwise, to republish, to post on servers or to redistribute to lists, requires prior specific permission and/or a fee.

SPAA'02, August 10-13, 2002, Winnipeg, Manitoba, Canada.

Copyright 2002 ACM 1-58113-529-7/02/0008 ...\$5.00.

\section{Categories and Subject Descriptors}

F.2.2 [Analysis of Algorithms and Problem Complexity]: Nonnumerical Algorithms and Problems; G.2.2 [Discrete Mathematics]: Graph Theory; G.2.3 [Discrete Mathematics]: Applications; J.2 [Computer Applications]: Physical Sciences and Engineering

\section{General Terms}

Algorithms

\section{Keywords}

Parallel computing, load balancing, algorithms, maximumflow, least squares

\section{INTRODUCTION}

In many applications of parallel computing, the distribution of data among processors implies a corresponding distribution of work. However, there are important exceptions to this rule that arise for one of two reasons. First, some portions of the data may be replicated on multiple processors, any of which could perform the associated work. Second, tasks may involve multiple data items which may not all reside on the same processor. Thus, all the interacting data will need to be combined on a single processor before the computation can be completed. In principle, any processor could perform this task (see, e.g. [9]), but for the purposes of this paper we will consider only those processors owning a portion of the relevant data-other options would increase the communication requirements.

Examples of such flexibly assignable work are common in scientific applications. In molecular dynamics simulations, a force is computed between any pair of particles that are close to each other. For large problems, these calculations are usually parallelized by dividing the particles among the processors [13]. If two close-by particles reside on different processors, then either processor could perform the computation. Kalé et al. [9] worked on heuristics to improve load balance by cleverly assigning such pairs in their molecular dynamics code, NAMD [10].

Another example arises in finite element simulations. These calculations consist of several computational phases, some of which are element based while others are node based. If, for instance, the mesh is partitioned so that processors own full elements, then nodes at the boundary between elements will be duplicated on at least two processors. Any of these pro- 
cessors could perform the node based operations for these shared nodes. If instead the mesh is partitioned by nodes, then some elements will be divided among multiple processors. Any of these processors could be employed to perform the element computation.

A third example comes from an important class of preconditioners known as overlapped Schwarz domain decomposition $[12,15]$. In this preconditioning scheme, processors perform one calculation on subdomains that overlap each other, and another calculation on disjoint subdomains. With the overlapped domains, some portions of the data are duplicated on multiple processors. Any of these processors could perform the calculations for these duplicated objects in the disjoint portion of the computation.

The freedom to assign work to any of several processors raises the question of how best to exploit this flexibility. In this paper we investigate using this freedom to improve load balance. That is, we want to give most of this flexibly assignable work to processors that would otherwise have too little to do. More formally, we address the following task assignment problem.

Given: A set of unit tasks and the (possibly singleton) set of processors that can perform them.

Find: An assignment of tasks to processors that minimizes the number of tasks assigned to the maximally loaded processor.

Despite its practical utility, to our knowledge, this problem has not been defined or addressed previously. After providing some basic definitions in $\S 2$, we investigate several combinatorial approaches to address the task assignment problem in $\S 3$. Some preliminary experimental results are included in an appendix, which provide evidence that significant gains in load balance can be achieved.

Besides formulating a new and practically important problem, this paper makes several technical contributions. First, we describe a parametric search solution that uses a standard maximum-flow solver as a probe function. This solution is simple to implement and allows for the use of any flow solver as a black box.

Our second combinatorial algorithm involves a more detailed analysis of the structure of the problem. Specifically, we devise a maximum-flow/minimum-cut theorem for our non-standard objective function. This result gives significant insight into the structure of the problem, and we use it to devise an augmenting path algorithm that mimics the structure of Ford-Fulkerson methods for maximum flows. It is also worth noting that our approach solves the problem of finding a maximum flow with the property that the largest flow on any terminal edge is minimized.

These combinatorial algorithms are sufficient for many problems in which a serial computation can determine the assignment as a preprocessing step to a parallel calculation. However, in some instances the characteristics of the parallel computation change over time, and the assignment must be recomputed. Our combinatorial methods are not particularly amenable to parallelization. For this reason, in $\S 4$ we present a continuous approximation to the problem that leads to a more easily parallelized numerical approach. The continuous approximation is closely related to the diffusion methodology widely employed for determining work transfers in dynamic load balancing [4]. We show that the flexibly assignable work problem can be formu- lated as a linearly-constrained optimization problem, i.e., $\min \|A x\|_{\infty}$, s.t. $B x=d$. Here, the linear constraints $B x=$ $d$ guarantee that assignment of tasks is valid, and $A x$ is the vector of processor loads. Minimizing the $\infty$-norm of this vector corresponds to minimizing the maximum processor load. Being a nonsmooth function, the $\infty$-norm is difficult to minimize. However, we show that in this context, minimizing the 2 -norm is sufficient to minimize the $\infty$-norm, which reduces the problem to the well-studied numerical kernel known as a linearly-constrained least squares problem. We then show that there are efficient parallel approaches to solve this problem. Of course, the discretized solution to the continuous approximation may not be identical to the actual solution to the discrete problem.

\section{PRELIMINARIES}

A flow network is defined by a directed graph $G=(V, E)$, with a source vertex $s$, a terminal vertex $t$, and a capacity for each edge $(i, j)$, which we denote by $c(i, j)$.

We will define a flow $f$ to be a function $f: E \rightarrow Z^{+}$ from edges to integers, and use $f(i, j)$ to denote the volume of flow along edge $(i, j)$. A flow must satisfy the capacity constraints on edges (i.e. $f(i, j) \leq c(i, j))$, and the flow conservation constraints

$$
\sum_{(i, k) \in E} f(i, k)=\sum_{(j, i) \in E} f(j, i) \text { for all } i \in V \backslash\{s, t\} .
$$

The value of a flow $|f|$ is defined by the flow leaving the source $s$,

$$
|f|=\sum_{(s, v) \in E} f(s, v) .
$$

A maximum flow (max-flow) is a flow that maximizes $|f|$.

A flow is complete if its value is equal to the cumulative capacity of edges leaving the source, i.e., $|f|=\sum_{(s, v) \in E} c(s, v)$. A graph that can support a complete flow will be called a complete-flow graph.

Given a graph $G$ and flow $f$, the residual graph $G_{f}$ has the same set of vertices as $G$ and all edges in $G$ (referred to here as forward edges), plus a matching set of backward edges that point in the opposite direction. The capacity of a forward edge is equal to its capacity in $G$ minus the flow assigned to that edge in $f$. The capacity of a backward edge is equal to the flow on the corresponding forward edge.

In the max-flow problem, an augmenting path is defined as a path from $s$ to $t$ along which more flow can be pushed. The capacity of a path is defined by the minimum of the capacities of its edges. Any path in $G_{f}$ from $s$ to $t$ with nonzero capacity is an augmenting path and can be used to increase the total flow.

Finding a maximum flow is a fundamental problem in combinatorial algorithms and has been the subject of numerous research efforts. Fundamentals of network flow algorithms can be found in $[2,16]$. In a more recent work, Goldberg and Rao give a history of maximum-flow bounds and relevant references [8].

The assignment of tasks to processors can be modeled as a flow on a network $G=(T, P, E)$, where each task is represented by a vertex in $T$, and each processor is represented by a vertex in $P$. All processor-vertices are connected to the terminal $t$ by terminal edges, and the source $s$ is connected to all the task-vertices by source edges. Task-vertices have assignment edges connecting them to all the processors, which 


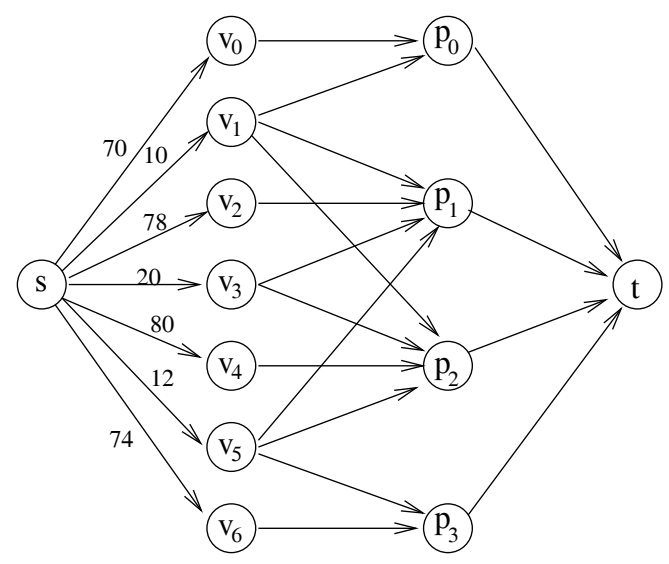

Figure 1: Example of an assignment graph.

the associated task can be assigned to. The graph can be simplified by combining all vertices that have identical sets of processor neighbors. We call such sets task groups. An example can be found in Figure 1.

The capacity of an edge from the source to a task-vertex is defined by the size of the corresponding task group. We will set the capacities for assignment edges and terminal edges to be infinite. We will call this graph an assignment graph. Notice that assignment graphs are complete-flow graphs.

Figure 1 illustrates an assignment graph. There are 7 task groups and 4 processors. $v_{1}$ corresponds to a task group of 10 tasks and can be assigned to processors $p_{0}, p_{1}$, and/or $p_{2}$. Notice that some of the task groups can be assigned to only one processor. This situation often arises in practice, and these tasks correspond to work that can be assigned only to a single processor.

We can consider the assignment of tasks as a flow from the task-vertices to processor-vertices. Our objective is to find a flow that assigns all the tasks to processors while minimizing the maximum load of any processor. We need the flow to be complete (full capacity of source edges is used) to guarantee assignment of all the tasks. We can define the flow problem as follows.

Given an assignment graph $G=(T, P, E)$, find a complete flow $f$ in $G$ that minimizes

$$
\max _{p \in P} f(p, t) .
$$

Although we define our flow problem for very specific flow graphs, the algorithms and analysis in the following sections are valid for any complete-flow graph. For a general graph, we would redefine $P$ in (1) to be the set of vertices that are connected to the terminal vertex (i.e., $P=\{v:(v, t) \in$ $E\})$. Thus, the problem we are solving is equivalent to that of finding a maximum flow that minimizes the largest flow along any terminal edge.

In Figure 2, a solution to an assignment problem is illustrated. Numbers on the edges correspond to flow assignments for these edges. This assignment gives an optimal solution where $p_{1}$ and $p_{2}$ are the maximally loaded processors with 89 tasks. Out of 20 tasks of task group $v_{3}, 11$ will be performed on $p_{1}$ and 9 will be performed on $p_{2}$.

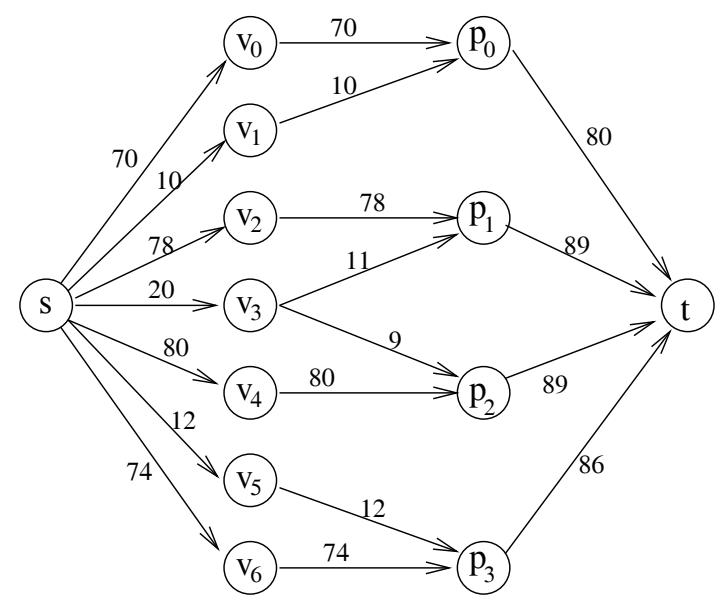

Figure 2: Example of a solution on an assignment graph.

\section{COMBINATORIAL SOLUTIONS}

As outlined in $\S 2$, assignment of tasks to processors can be formulated as a flow in a network. In this section, we will investigate the relation between classical flow problems and the task assignment problem. First, we will discuss parametric search solutions that use standard max-flow techniques as a probe function, and describe a polynomial time algorithm for the task assignment problem. Then we will show how Ford-Fulkerson methods can be used to solve our problem. Specifically, we will revise the definition of augmenting paths and cuts, and show that any maximum-flow algorithm based on the Ford-Fulkerson method can be used for the task assignment problem.

\subsection{Parametric Search}

A parametric search algorithm has two components: a probe function that determines whether there is a solution with a cost less than a specified value, and a method to search on the space of candidate optimal solution values. Below, we first show how standard maximum-flow algorithms can be used as a probe function for our problem. Our cost function is the maximum work assigned to any single processor. Then we discuss two techniques to search the space of candidate values. The following lemma formalizes our claim for maximum-flow algorithms being used as a probe function.

LEMMA 3.1. There is a solution to the task assignment problem with cost $\leq B$ if and only if there exists a complete flow on the modified graph where all terminal edges have capacity $B$.

Proof. Construct the assignment graph as described in $\S 2$ and change the capacities of all terminal edges to $B$. We claim that there is a solution to the task assignment problem with cost $\leq B$ if and only if the maximum flow uses the capacity of all the source edges. Proof of this claim follows. Sufficiency. Bounds on capacities of terminal edges guarantee that no processor is assigned more than $B$ units of work, and if a flow uses all the source edge capacity then all work is assigned to processors. Moreover, the flow solution provides the corresponding task assignments. 
Necessity. Assume there is a solution to the task assignment problem where no processor is assigned more than $B$ units of work. We can use the assignments of tasks in this solution to find a corresponding flow solution.

To solve the task assignment problem we must find the minimal value of $B$ for which a max-flow solution uses all the source edge capacities. We present two algorithms for finding this value in the following two subsections. The complexity of the first one is pseudo-polynomial, whereas the second one is polynomial. The relative performance of these algorithms depends on problem specific details. Bisection search is generally more effective when searching over a large space, whereas incremental search is more effective in a more localized search space.

\subsubsection{Bisection Search}

Bisection search is a standard technique used in parametric search algorithms. It starts with a lower and an upper bound on the optimal solution value, and discards half of the interval by probing on the midpoint of the current bounds. This gives an $\epsilon$-approximation algorithm for real-valued solutions, but finds an exact solution when the optimal solution value is an integer, as in the case of our problem.

For the task assignment problem, the total number of tasks is an upper bound on the cost of an assignment, and the number of tasks divided by $|P|$ is a lower bound. Thus, a bisection search gives the following result.

Theorem 3.2. If $W_{T}$ is the total number of tasks, bisection search solves the task assignment problem optimally with $O\left(\log W_{T}\right)$ probe calls.

\subsubsection{Incremental Search}

An incremental search starts with a lower bound for the optimal solution value and increases it until the optimal value is found. The increments should be small to avoid missing the optimal value, but large for efficiency. The following lemma and theorem show how the lower bound can be increased after a failed probe call - that is, a max-flow problem with terminal edge capacities $B$ in which not all the source edge capacity is utilized.

LEMMA 3.3. Let $(u, t)$ be a terminal edge that is not saturated in a maximum-flow solution $f$ for a probe value $B$ (i.e., $f(u, t)<c(u, t)=B)$. Then for any probe value $B^{\prime}>B$, there is a maximum-flow solution $f^{\prime}$ in which $f^{\prime}(u, t) \leq f(u, t)$.

Proof. When the Ford-Fulkerson method is used to achieve an optimal solution $f^{\prime}$ for bound $B^{\prime}>B$ by using $f$ as an initial solution, we can get an optimal solution with $f^{\prime}(u, t) \leq f(u, t)$. First, note that $u$ is not reachable from $s$ in $G_{f}$, and increasing terminal edge capacities does not make $u$ reachable from $s$. Furthermore, $u$ will not be reachable while the flow is being modified via augmenting paths. Consider the first augmenting path that will add a vertex to the set of vertices $u$ is reachable from. Observe that such a path should reach a vertex that can reach to $u$, which contradicts $u$ 's non-reachability from $s$.
THEOREM 3.4. For a failed probe with terminal edge capacity $B$, let $W_{r}>0$ be the total unused source edge capacity, and let $K$ be the number of saturated terminal edges. Then there is no feasible solution to the task assignment problem with cost less than $B+W_{r} / K$.

Proof. By the result of Lemma 3.3, additional flow cannot go to any of the unsaturated terminal edges. In the best case, additional flow will be equally distributed among the set of saturated terminal edges.

As a result of Theorem 3.4, a failed probe value $B$ can be increased to $B+\frac{W_{r}}{K}$, as exploited in Algorithm IncSearch.

Algorithm IncSearch

$B \leftarrow W_{T} /|P|$

while not $\operatorname{Probe}(B)$

Let $K$ be the number of saturated terminal edges,

and $W_{r}$ be the total unused source edge capacity.

$B \leftarrow B+\frac{W_{r}}{K}$

return $B$;

The following lemma proves that Algorithm IncSearch terminates and gives a bound on the number of probes it makes.

Lemma 3.5. Algorithm IncSearch terminates after at most $|P|$ probes.

Proof. When a probe value is increased, if all previously saturated edges remain saturated then the probe call will succeed. Thus, when a probe fails, at least one new terminal edge is not saturated. That is, each failed probe decreases the number of saturated terminal edges by at least one.

TheOREM 3.6. Algorithm IncSearch finds an optimal solution and makes $O(|P|)$ probes.

Proof. We start with a lower bound. According to Theorem 3.4, increases on $B$ are minimal. Thus we do not miss the optimal value. Lemma 3.5 ensures that the algorithm terminates after $O(|P|)$ probes with an optimal solution.

Notice that successive probes solve max-flow problems on the same graph in an incremental manner, where only the capacities of the terminal edges increase. Thus the previous flow solution gives a feasible solution (though not optimal) for the next flow solution, which might be exploited for efficiency. Using Ford-Fulkerson method in its simplest way will give a complexity of $O\left(W_{T} *|E|\right)$ for all the probes, and thus for the algorithm.

\subsection{Ford-Fulkerson Method}

The Ford-Fulkerson method has been the basis of a number of algorithms to solve the max-flow problem. It is built on three basic concepts: residual graphs, augmenting paths, and cuts [2]. In this section, we will discuss how it can be adopted to the task assignment problem. First, we will revise the definitions of augmenting paths and cuts for the task assignment problem, then state and prove a version of the maximum-flow/minimum-cut theorem for the task assignment problem. The result will enable any algorithm 
based on the Ford-Fulkerson method to be used to solve our problem.

The generic Ford-Fulkerson method starts with a zero flow and continues to add to the flow along augmenting paths until no augmenting paths are left. In the task assignment problem, we will use augmenting paths to shift flow (tasks) from a maximally loaded terminal edge (processor) to a less loaded terminal edge. Formally, an augmenting path $(p t, u, v)$ is a path $p t$ in $G_{f}$ that starts with the vertex $u$ of a maximally loaded terminal edge $(u, t)$, ends at the vertex $v$ of a less-loaded terminal edge $(v, t)$, and does not go through $t$. We define the capacity $c(p t, u, v)$ of an augmenting path to be the minimum of the capacities of its edges and half of the difference between the flow on the first and last terminal edges, rounded down to an integer,

$c(p t, u, v)=\min \left(\left\lfloor\frac{f(u, t)-f(v, t)}{2}\right\rfloor, \min \left\{c_{f}(i, j):(i, j)\right.\right.$ on $\left.\left.p t\right\}\right)$.

This implies that the capacity of a path between two processors whose loads differ by just one is zero, since such an augmentation will not yield a more balanced distribution.

We can update flow assignment in the graph for edges on the path and the two terminal edges connecting processorvertices to the terminal to obtain a more balanced distribution, as stated by the following lemma.

LEMMA 3.7. Let $f$ be the current flow, pt be an augmenting path, and $c>0$ be the capacity of this path. Define $f^{+}(i, j)$ for all edges as

$$
f^{+}(i, j)= \begin{cases}f(u, t)-c & \text { if } i=u, j=t \\ f(v, t)+c & \text { if } i=v, j=t \\ f(i, j)+c & \text { if }(i, j) \text { is on } p t \\ f(i, j)-c & \text { if }(j, i) \text { is on } p t \\ f(i, j) & \text { otherwise }\end{cases}
$$

Then $f^{+}$does not change the total flow (i.e., $\left|f^{+}\right|=|f|$ ), but decreases either the maximum load or the number of maximally loaded terminal edges.

Proof. For the total flow to change we must decrease flow from $s$. This is possible only if there is an edge $(v, s)$ in $p t$, but this edge must be followed by another edge $(s, u)$, and thus total flow from $s$ does not change. With the same argument, flow conservation constraints are satisfied for all vertices. Since $p t$ does not go through $t$, augmentation will affect only two terminal edges: $(u, t)$ and $(v, t)$. By definition of an augmenting path, $(u, t)$ is a maximally loaded terminal edge, and we decrease its load. By definition of the capacity of an augmenting path, the load of $(v, t)$ cannot be as high as $(u, t)$, after we increase it.

In traditional flow problems, a cut $(S, T)$ in $G$ is defined as a partition of vertices into $S$ and $T$ in which $s \in S$ and $t \in T$. The cost of a cut is defined as the sum of capacities of edges from $S$ to $T$. The cost of a minimum cut and value of a maximum flow are equal. A minimum cut corresponds to a bottleneck in the flow from source to terminal. For the task assignment problem, we will define a cut as a bipartitioning $\left(P_{1}, P_{2}\right)$ of the processor-vertices. The cost of a cut is defined to be the maximum load in $P_{1}$ when

(i) processors in $P_{1}$ are equally loaded, (ii) all the tasks that can be assigned to a processor in $P_{2}$ are assigned to processors in $P_{2}$.

By "equally loaded" we mean that the loads of any two processors differ by at most one. Cuts will help to identify a bottleneck in the problem, just as in maximum flow problems. A bottleneck in our problem is a group of processors that have to perform a large set of tasks. Unlike the maximum flow problem, cuts provide lower bounds on the cost.

With the above definitions, we have the following maximumflow/minimum-cut theorem for the task assignment problem.

THEOREM 3.8. The following statements are equivalent:

1. Flow $f$ minimizes the load of the maximally loaded processor.

2. There is no augmenting path that decreases the maximum load in $G_{f}$.

3. The maximum load is equal to the cost of a cut $\left(P_{1}, P_{2}\right)$.

Proof. $\quad-(1) \Longrightarrow(2)$ Assume the contrary, that there exists an augmenting path to decrease the maximum load. Then we can use this path to decrease the maximum load, so $f$ is not an optimal flow.

- $(2) \Longrightarrow(3)$ Let $P_{1}$ be the set of processors with maximum load plus processors reachable from a maximally loaded processor in $G_{f}$. The set $P_{2}$ contains the remaining processors. By construction, there are no augmenting paths from $P_{1}$ to $P_{2}$. This guarantees that all tasks between $P_{1}$ and $P_{2}$ are assigned to $P_{2}$ processors. Also, since there are no augmenting paths in $G_{f}$, the loads of all processors in $P_{1}$ are either equal to or one less than the maximum load. That is, the processors in $P_{1}$ are equally loaded.

- $(3) \Longrightarrow(1)$ Since all tasks which could be assigned to either $P_{1}$ or $P_{2}$ are assigned to processors in $P_{2}$, the work currently assigned to processors in $P_{1}$ must be performed by processors in $P_{1}$. The best we can do is to assign all work equally, which is guaranteed by the first condition in the definition of a cut, so $f$ is an optimal solution.

COROllary 3.9. Any algorithm based on the Ford-Fulkerson method can be used to solve the task assignment problem.

It is worth noting that although any algorithm based on the Ford-Fulkerson method might be used to solve this problem optimally, the complexity results might vary from those of the conventional max-flow problem.

Below, we present an algorithm AugPath, which finds an optimal solution using augmenting paths.

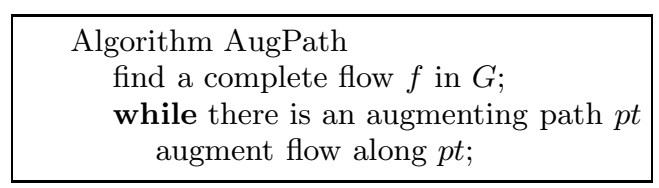


Theorem 3.10. Algorithm AugPath finds an optimal solution in $O\left(|E| * \log |P| * W_{T}\right)$-time.

Proof. Correctness of the algorithm is implied by Corollary 3.9. Finding an augmenting path takes $O(E)$-time in the worst case. By Lemma 3.7, each augmenting path either decreases the maximal load or the number of maximally loaded processors. This gives a loose $|P| * W_{T}$ bound on the number of augmenting paths required. A better bound is possible however. When the maximum load is in the range $\left[W_{T} / 2, W_{T}\right]$, only one processor might have the maximum value, and thus each augmenting path will decrease the maximum load by one. Generally, when the maximum is in the range $\left[W_{T} /(k+1), W_{T} / k\right]$ there can be at most $k$ processors with the maximum load and $k$ augmenting paths may be needed to decrease the maximum load. So the total number of augmenting paths can computed as

$$
\begin{aligned}
& \sum_{1 \leq k \leq|P|-1}\left(\frac{W_{T}}{k}-\frac{W_{T}}{k+1}\right) k \\
= & W_{T} \sum_{1 \leq k \leq|P|-1}\left(\frac{k+1-k}{k(k+1)}\right) k \\
= & W_{T} \sum_{1 \leq k \leq|P|-1} \frac{1}{k+1} \\
= & O\left(W_{T} * \log |P|\right) .
\end{aligned}
$$

\section{NUMERICAL SOLUTION}

The flow formulations described above provide efficient, serial algorithms for optimizing task assignments. Unfortunately, flow algorithms are difficult to parallelize, particularly for large numbers of processors. In this section we describe a continuous version of the problem and show that it reduces to a well-studied numerical computation. Although this approach does not provide an integral solution, its parallelizability may make it preferable for many applications. In spirit, our approach is similar to the widely used diffusion methods to determine how much work to transfer between processors in dynamic load balancing $[4,6,7]$.

In our numerical formulation, each task group generates an equation. Say the task group has $m$ tasks in it. If the task group can be assigned to any of $k$ processors, then there will be $k$ unknowns associated with the task group. Each of these unknowns $x_{1}, \ldots, x_{k}$ encodes the assignment of the corresponding task group to one of the processors. In a discrete formulation we would want the $x_{i}$ values to be integral, but in our continuous formulation we impose the following, weaker, set of equality and inequality constraints.

$$
\sum_{i=1}^{k} x_{i}=m, \text { and } x_{i} \geq 0 \text { for all } 1 \leq i \leq k
$$

The $x$ values can be used to encode the work assigned to each of the $|P|$ processors. The task group with $k$ potential processors will generate $k$ columns of length $|P|$. The $i$ th column is all 0's except for a single 1 in the row number that corresponds to the processor associated with $x_{i}$. These columns can be treated as a matrix, and multiplying the $x$ vector by this matrix gives a $|P|$ length vector containing the work assigned to each of the processors.

We can continue this construction, adding variables, constraints, and work contributions from all $|T|$ tasks. Letting $|Q|$ denote the sum over all tasks of the number of processors that task could be assigned to, we obtain the following problem.

$$
\min _{x}\|A x\|_{\infty} \text { subject to } B x=d \text { and } x \geq 0,
$$

where $A$ is $|P| \times|Q|, B$ is $|T| \times|Q|$, and both have only a single 1 in each column. In the flow terminology from $\S 2$, the $x$ vector is the assignment of a (possibly fractional) flow to the edges from source-adjacent nodes to terminal-adjacent nodes (see Figure 1). $A x$ is the amount of flow into each terminal-adjacent node, $B x$ is the flow out of each sourceadjacent node and $d$ is the vector of sizes of task groups. So $B x=d$ merely encodes the flow preservation property for each source-adjacent node in a complete flow. The $\infty$-norm reflects our desire to minimize the work of the maximally loaded processor. It is worth remarking that tasks that can be performed only by a single processor can be removed from the variable set, reducing the size of the problem. For the example from Figure 1, we get the following matrices in our numerical formulation.

$$
A=\left(\begin{array}{llllllllllll}
1 & 1 & & & & & & & & & & \\
& & 1 & & 1 & 1 & & & 1 & & & \\
& & & 1 & & & 1 & 1 & & 1 & & \\
& & & & & & & & & 1 & 1
\end{array}\right)
$$

$$
B=\left(\begin{array}{ccccccccccccc}
1 & & & & & & & & & & & \\
& 1 & 1 & 1 & & & & & & & & \\
& & & & 1 & & & & & & & \\
& & & & & 1 & 1 & & & & & \\
& & & & & & & 1 & & & & \\
& & & & & & & & 1 & 1 & 1 & \\
& & & & & & & & & & & 1
\end{array}\right) \quad d=\left(\begin{array}{c}
70 \\
10 \\
78 \\
20 \\
80 \\
12 \\
74
\end{array}\right)
$$

Unfortunately, the $\infty$-norm is not smooth and so can be difficult to minimize. A similar problem arises in the diffusion approach where a 1-norm is approximated by the smooth alternative of a 2-norm. We will similarly choose to replace our $\infty$-norm with a 2 -norm as follows.

$$
\min _{x}\|A x\|_{2} \text { subject to } B x=d \text { and } x \geq 0
$$

This formulation is an instance of what is known as a linearlyconstrained least squares problem. Unlike the diffusion methodology, in our case we lose nothing by transforming to a smoother approximation since for our problem a solution to the 2 -norm problem also solves the $\infty$-norm problem. This is shown by the following theorem.

Theorem 4.1. Any $x$ that solves Problem (3) is also a solution for Problem (2).

Proof. Begin by noting that Problem (2) is identical to the flow problem from $\S 3$, except that variables are continuous instead of discrete. The analysis from $\S 3$ generalizes to continuous variables in a straightforward manner, so the structure of a solution to Problem (2) is the same as the structure of a solution to the flow formulation. Specifically, 
a subset of processors $\left(P_{1}\right)$ will have the same maximum load $\left(l_{\max }\right)$, and all tasks that can be assigned to processors with smaller loads $\left(P_{2}\right)$ have been so assigned.

Now let $x$ be a solution to Problem (3). This problem has the same constraints as Problem (2), so it shares the same space of feasible solutions. As with the solution to Problem (2), the processors in $P_{1}$ will need to be assigned at least a total of $\left|P_{1}\right| l_{\max }$ load. It is easy to show that the contribution of these processors to the 2-norm is minimized when all their loads are equal. Any transfer of work from processors in $P_{2}$ to processors in $P_{1}$ will only increase the 2norm. So a solution to Problem (3) must also be a solution to Problem (2).

Least squares problems are fundamental to linear algebra (see, e.g.. [1]). Constrained least squares problems have been studied by several authors. Of interest to us are iterative methods that are amenable to parallelization. Note that since we are using the continuous formulation as an approximation to a discrete problem, a low accuracy numerical solution is sufficient.

One way to deal with linear equality constraints is the method of weighting [1]. This method moves the equality constraints into the objective function, but severely penalizes slack in these rows by weighting these equations. So Problem (3) is transformed to the form

$$
\min _{x}\left\|\left(\begin{array}{c}
\gamma B \\
A
\end{array}\right) x-\left(\begin{array}{c}
\gamma d \\
0
\end{array}\right)\right\|_{2} \text { subject to } x \geq 0
$$

where $\gamma$ is a large number used to penalize slack in the equality constraints. This transforms our problem to an instance of a nonnegative least squares problem,

$$
\min _{x}\|C x-b\|_{2} \text { subject to } x \geq 0 .
$$

The nonnegative least squares problem is equivalent to the following optimization problem.

$$
\min _{x} \frac{1}{2} x^{T} E x-c^{T} x \text { subject to } x \geq 0,
$$

where $E=C^{T} C$ and $c=C^{T} b$. Cryer proposed the following SOR iteration for solving nonnegative least squares problems [3],

$x_{i}^{k+1}=\max \left\{0, x_{i}^{k}-\frac{\omega}{e_{i i}}\left(c_{i}-\sum_{j<i} e_{i j} x_{j}^{k+1}-\sum_{j \geq i} e_{i j} x_{j}^{k}\right)\right\}$.

This is a standard stencil operation and requires only local communication with neighbor processors. Hence, it is amenable to efficient parallelization.

The least squares technique will give a non-integral solution, which needs to be discretized for task assignment. The continuous solution can be easily mapped to a feasible solution by adjusting the assignments for each task group. Consider a group of tasks that can be assigned to either $p_{0}$ or $p_{1}$. We can round the total assignment to $p_{0}$ up to an integer, and assign that many tasks to $p_{0}$ and assign the remainder to $p_{1}$. Notice that this adjustment has only local effects and is easy to generalize for more processors. We do not have any bounds on the impact of such rounding operations on solution quality.
Table 1: Results on molecular dynamics problems.

\begin{tabular}{l|c|c||r|r|r}
\hline Problem & $\mathrm{N}$ & $\mathrm{F}$ & $\mathrm{P}$ & Init. & Imp. \\
\hline membrane & 7134 & 4052598 & 8 & 5.80 & 0.32 \\
& & & 64 & 21.17 & 0.21 \\
& & & 512 & 58.34 & 2.16 \\
\hline mixer.half.1 & 4818 & 17220 & 8 & 121.55 & 110.78 \\
& & & 64 & 163.70 & 134.07 \\
\hline mixer.half.2 & 4818 & 17360 & 8 & 146.48 & 130.40 \\
& & & 64 & 195.59 & 155.15 \\
\hline
\end{tabular}

\section{EXPERIMENTAL RESULTS}

We have applied our techniques to problems from two application domains: molecular dynamics and overlapped domain decomposition. In each case, as described in $\S 4$ we solved the least squares formulation of the problem in serial, and used Gauss-Seidel iterations [5] to generate a new distribution of work among processors. We define load imbalance as $(\max -a v g) * 100 / a v g$, where $\max$ and $a v g$ denote the maximum and average processor load, respectively.

For the molecular dynamics application, we used data provided by Plimpton which came from his spatial decomposition code [13]. In a molecular dynamics simulation, the work is dominated by the number of forces that need to be computed between pairs of nearby atoms. In this code, a bounding box encloses all the atoms, the box is divided into $P$ regions of equal volume and each of $P$ processors is responsible for atoms residing within one of the boxes. Flexibility arises when two atoms belonging to different processors are close enough to interact. Larger interaction cutoffs and smaller regions each increase the fraction of flexibly-assignable work. If the atoms are uniformly distributed through the bounding box (e.g. for simulations with periodic boundaries), then the load will generally be well balanced. But for problems in which the atom distributions are inhomogeneous, significant load imbalance can arise.

We present results for two types of problems in Table 1. In this table $N$ represents the number of particles and $F$ is the total number of pairwise force computations. A simple way to partition a set of flexibly assignable force computations is to assign half to each of the two processors they span. The load imbalance induced by this strategy is detailed in column 'Init.' in the table. Column 'Imp.' contains the load imbalance resulting from using our least squares algorithm to assign work. Clearly, our approach can result in a significant reduction in load imbalance. It is worth noting that these are problems of modest size, and so do not require very large numbers of processors.

Of the problems in Table 1, the first comes from a biological simulation of a membrane, in which the atomic densities are higher within the membrane than within the surrounding fluid. For large numbers of processors we are able to reduce the load imbalance from an initial $58 \%$ to just over $2 \%$. The next two problems are instances of a simulation of a rotating drum being used to mix solid particles as described in [14]. The particles fill only a fraction of the volume of the drum, leading to significant load imbalance. Specifically, some of the processors are responsible for regions of space that have few or no particles. For this problem, the particles are treated as rigid bodies, and so the cutoff distances are very short. As a consequence, there are few flexibly 
assignable interactions, which limits our ability to improve load balance. Despite these inherent difficulties, we are still able to significantly improve the overall load balance.

The second data set comes from an important class of preconditioners known as overlapped Schwarz domain decomposition $[12,15]$. In this preconditioning scheme, processors perform one phase of the calculation on subdomains that overlap each other, and another phase on disjoint subdomains. To achieve high performance it is important to balance the load of each phase. To accomplish this, we first choose a partition for which the overlapped subdomains are balanced [12]. Such a partition does not necessarily provide load balance for the disjoint phase as evidenced by the "Initial" column in Table 2. To improve the load balance, we can then exploit flexibility in task assignment for the disjoint phase. Specifically, overlapped portions of the initial decomposition correspond to duplicated data, and any processor owning those data can perform the associated task in the disjoint phase. Table 2 displays our experimental results on a set of sparse test matrices arising from applications where overlapped subdomain preconditioners are used [12]. In this table, $N$ denotes the number of rows and columns, and $N N Z$ is the number of nonzeros in the matrix, and $P$ is the number of processors. These are all modest sized problems and so do not require a very large number of processors. The column labeled 'Initial' describes the load imbalance associated with assigning the disjoint subdomains to processors and then generating the overlapped partitions by enlarging the disjoint domains by one layer. The column labeled 'Improved' details the load imbalance resulting from our least squares solution. The results indicate that significant improvements in load balance are achieved by our techniques.

It is worth noting that each Gauss-Seidel iteration consists of a simple traversal of tasks, and the algorithm converges in a few iterations. The load imbalance decreases sharply in the first few iterations, and then levels off. In most instances, the algorithm terminated after 3 or 4 iterations.

\section{CONCLUDING REMARKS}

We have posed and addressed the problem of distributing flexibly assignable work to processors to minimize load imbalance. This paper considers the problem in a general form, whereas exploiting problem-specific information might yield more efficient solutions. For instance, in the molecular dynamics application and in many other cases each task can be assigned to one of at most two processors. We can exploit this fact to formulate the problem as a bounded least squares problem, $\min \|A x+b\|_{2}$ s.t. $0 \leq x \leq u$, where $u$ is a vector of upper bounds on decision variables. This formulation grants simpler and more efficient solution techniques than the more general linearly-constrained least squares formulation.

Although we restrict our discussions in this abstract to homogeneous parallel computers, our techniques can also be generalized to address heterogeneous systems as well. Details can be found in [11].

This paper introduced a novel way to improve load balance, and suggests several research directions. First, the structure of this problem may allow specialization of flow techniques. It will be interesting to investigate if and how the advanced techniques for max-flow problems can be suited to our problem for more efficient combinatorial algorithms.
Table 2: Results for the overlapped subdomain preconditioners

\begin{tabular}{l|rr||r|rr}
\hline Matrix & $N$ & $N N Z$ & $P$ & Initial & Improved \\
\hline Braze & 1344 & 142296 & 4 & 1.25 & 0.00 \\
& & & 8 & 31.52 & 0.34 \\
& & & 16 & 77.25 & 1.36 \\
& & & 32 & 126.47 & 43.16 \\
\hline Defroll & 6001 & 173718 & 4 & 13.28 & 0.08 \\
& & & 8 & 25.47 & 7.28 \\
& & & 16 & 17.89 & 5.86 \\
& & & 32 & 41.66 & 15.94 \\
\hline DIE3D & 9873 & \multirow{2}{*}{1723498} & 4 & 20.60 & 0.13 \\
& & & 8 & 28.52 & 0.19 \\
& & & 16 & 91.39 & 2.50 \\
& & & 32 & 138.07 & 9.08 \\
\hline dday & 21180 & \multirow{2}{*}{1033324} & 4 & 1.52 & 0.14 \\
& & & 8 & 3.59 & 0.07 \\
& & & 16 & 13.08 & 0.55 \\
& & & 32 & 18.26 & 0.75 \\
\hline visco & 23439 & \multirow{2}{*}{1136966} & 4 & 16.28 & 0.30 \\
& & & 8 & 34.19 & 1.77 \\
& & & 16 & 53.11 & 3.41 \\
& & & 32 & 84.49 & 5.52 \\
\hline sls & 36771 & 2702280 & 4 & 2.28 & 0.15 \\
& & & 8 & 14.36 & 0.01 \\
& & & 16 & 16.88 & 0.07 \\
& & & 32 & 29.74 & 0.80 \\
\hline ocean & 143437 & \multirow{2}{*}{819186} & 4 & 4.92 & 0.21 \\
& & & 8 & 9.22 & 1.43 \\
& & & 16 & 17.39 & 3.35 \\
& & & 32 & 41.61 & 6.15 \\
\hline
\end{tabular}

Second, it would be helpful to generalize these techniques for non-unit tasks. Although the general problem corresponds to number partitioning, one can look at special cases like Cartesian partitions as in the case of molecular dynamics applications. Finally, using these techniques in different applications will be interesting. We keep identifying new sources of flexibly-assignable tasks, where our techniques can be used to improve load balance.

\section{REFERENCES}

[1] BJÖRCK, A. Numerical Methods for Least Squares Problems. SIAM, 1996.

[2] Cormen, T. H., Leiserson, C. E., And Rivest, R. L. Introduction to Algorithms. MIT Press and McGraw-Hill, Cambridge, MA, 1990.

[3] CRYer, C. The solution of a quadratic programming problem using systematic overrelaxation. SIAM J. Control and Optimization 9 (1971), 385-392.

[4] Cybenko, G. Dynamic load balancing for distributed memory multiprocessors. J. Parallel Distrib. Comput. 7 (1989), 279-301.

[5] Dax, A. Bounded least squares problem. ACM Trans. Math. Software (1991).

[6] Diekmann, R., Frommer, A., and Monien, B. Efficient schemes for nearest neighbor load balancing. Parallel Comput. (1999), 789-812.

[7] Elsässer, R., Monien, B., And Preis, R. Diffusive load balancing schemes on heterogeneous networks. In 
Proc. 12th ACM Symp. Parallel Alg. Arch. (SPAA) (2000), pp. 30-38.

[8] Goldberg, A. V., And RaO, S. Beyond the flow decomposition barrier. J. ACM 45 (1998), 783-797.

[9] Kalé, L., Bhandarkar, M., and Brunner, R. Load balancing in parallel molecular dynamics. In Fifth Intl. Symp. Solving Irregularly Structured Problems in Parallel (1998), vol. 1457 of Lecture Notes in Computer Science, Springer-Verlag, pp. 251-262.

[10] Kalé, L., Skeel, R., Bhandarkar, M., Brunner, R., Gursoy, A., Krawetz, N., Phillips, J., Shinozaki, A., Varadarajan, K., And Schulten, K. NAMD2: Greater scalability for parallel molecular dynamics. J. Comp. Phys. 15 (1999), 283-312.

[11] Pinar, A. Combinatorial Algorithms in Scientific Computing. PhD thesis, Dept. of Computer Science, UIUC, 2001.

[12] Pinar, A., and Hendrickson, B. Partitioning for complex objectives. In Proc. Intl. Parallel 86 Distrib. Processing Symp. (2001).

[13] Plimpton, S. Fast parallel algorithms for short-range molecular dynamics. J. Comp. Phys. 117 (1995), 1-19.

[14] Silbert, L., Ertas, D., Grest, G., Halsey, T., Levine, D., And Plimpton, S. J. Granular flow down an inclined plane: Bagnold scaling and rheology. Phys. Rev. E 64 (2001), 51302.

[15] Smith, B., Buørstad, P., And Gropp, W. Domain Decomposition: Parallel Multilevel Methods for Elliptic Partial Differential Equations. Cambridge University Press, Cambridge, UK, 1996.

[16] Tarjan, R. E. Data Structures and Network Algorithms. SIAM, 1983. 